\title{
A bisymmetry equation on restricted domain
}

\author{
By IMRE KOCSIS (Debrecen)
}

July 26, 2006

\begin{abstract}
Let $X \subset \mathbb{R}$ be an interval of positive length and define the set $\Delta$ by

$$
\Delta=\{(x, y) \in X \times X \mid x \geq y\} .
$$

In this note we give the solution of the equation

$$
F(G(x, y), G(u, v))=G(F(x, u), F(y, v)),
$$

which holds for all $(x, y) \in \Delta$ and $(u, v) \in \Delta$, where the functions $F$ : $X \times X \rightarrow X$ and $G: \Delta \rightarrow X$ are continuous and strictly increasing in each variable and $F$ is symmetric.
\end{abstract}

Mathematics Subject Classification (2000). 39B22.

Key words and phrases. bisymmetry, restricted domain.

\section{Introduction}

In the following we denote the set of real numbers and positive integers by $\mathbb{R}$ and $\mathbb{N}$, respectively. By an interval we mean a subinterval of positive length of $\mathbb{R}$ (possibly unbounded) and by a rectangle we mean the Cartesian product of two intervals. A real-valued function is called a $C M$ function if it is defined on an interval or on a rectangle and it is continuous and strictly monotonic (not necessary in the same sense) in each variable.

Let $X$ and $Y$ be intervals, $R$ be a rectangle such that $X \times Y \subset R$. A function $Q: R \rightarrow \mathbb{R}$ is a quasisum on the rectangle $X \times Y$ if there exist $C M$ functions $\alpha: X \rightarrow \mathbb{R}, \beta: Y \rightarrow \mathbb{R}$ and $\gamma: \alpha(X)+\beta(Y) \rightarrow \mathbb{R}$ such that $Q(x, y)=\gamma(\alpha(x)+\beta(y))$ for all $(x, y) \in X \times Y$. In particular if $X=Y$ and $Q$ is symmetric we can assume that $\alpha=\beta$. (It follows from the symmetry of $Q$ that $\alpha(x)+\beta(y)=\alpha(y)+\beta(x),(x, y) \in X^{2}$. Thus there exists $c \in \mathbb{R}$ such that $\beta(x)=\alpha(x)+c, x \in X$, which implies that $Q(x, y)=\gamma(\alpha(x)+\alpha(y)+c)=$ $\gamma_{1}(\alpha(x)+\alpha(y))$, where $\left.\gamma_{1}(t)=\gamma(t+c)\right)$.

The equation of generalized bisymmetry

$$
F\left(G_{1}(x, y), G_{2}(u, v)\right)=G\left(F_{1}(x, u), F_{2}(y, v)\right)
$$


where the functions are defined on rectangles was investigated by several authors (see e.g. Aczél [1] and Maksa [3]). The general $C M$ solution of equation (B) is given by the following

Theorem 1. (Maksa [3]) Let $X_{11}, X_{12}, X_{21}$, and $X_{22}$ be intervals of positive length, and let $F_{1}: X_{11} \times X_{12} \rightarrow \mathbb{R}, F_{2}: X_{21} \times X_{22} \rightarrow \mathbb{R}, G_{1}: X_{11} \times$ $X_{21} \rightarrow \mathbb{R}, G_{2}: X_{12} \times X_{22} \rightarrow \mathbb{R}, F: G_{1}\left(X_{11}, X_{21}\right) \times G_{2}\left(X_{12}, X_{22}\right) \rightarrow \mathbb{R}, G:$ $F_{1}\left(X_{11}, X_{12}\right) \times F_{2}\left(X_{21}, X_{22}\right) \rightarrow \mathbb{R}$ be $C M$ functions. Equation (B) holds for all $(x, y, u, v) \in X_{11} \times X_{21} \times X_{12} \times X_{22}$ if, and only if, there exists an interval $I$ and there exist $C M$ functions $\varphi: I \rightarrow \mathbb{R}, \alpha_{1}: G_{1}\left(X_{11}, X_{21}\right) \rightarrow \mathbb{R}, \alpha_{2}$ : $G_{2}\left(X_{12}, X_{22}\right) \rightarrow \mathbb{R}, \gamma_{1}: F_{1}\left(X_{11}, X_{12}\right) \rightarrow \mathbb{R}, \gamma_{2}: F_{2}\left(X_{21}, X_{22}\right) \rightarrow \mathbb{R}, \beta_{11}: X_{11} \rightarrow$ $\mathbb{R}, \beta_{12}: X_{12} \rightarrow \mathbb{R}, \beta_{21}: X_{21} \rightarrow \mathbb{R}$, and $\beta_{22}: X_{22} \rightarrow \mathbb{R}$ such that

$$
\begin{array}{ll}
F(x, y)=\varphi^{-1}\left(\alpha_{1}(x)+\alpha_{2}(y)\right), & (x, y) \in G_{1}\left(X_{11}, X_{21}\right) \times G_{2}\left(X_{12}, X_{22}\right) \\
F_{1}(x, y)=\gamma_{1}^{-1}\left(\beta_{11}(x)+\beta_{12}(y)\right), & (x, y) \in X_{11} \times X_{12} \\
F_{2}(x, y)=\gamma_{2}^{-1}\left(\beta_{21}(x)+\beta_{22}(y)\right), & (x, y) \in X_{21} \times X_{22} \\
G(x, y)=\varphi^{-1}\left(\gamma_{1}(x)+\gamma_{2}(y)\right), & (x, y) \in F_{1}\left(X_{11}, X_{12}\right) \times F_{2}\left(X_{21}, X_{22}\right) \\
G_{1}(x, y)=\alpha_{1}^{-1}\left(\beta_{11}(x)+\beta_{21}(y)\right), & (x, y) \in X_{11} \times X_{21} \\
G_{2}(x, y)=\alpha_{2}^{-1}\left(\beta_{12}(x)+\beta_{22}(y)\right), & (x, y) \in X_{12} \times X_{22} .
\end{array}
$$

The following question was published as an open problem by R. Duncan Luce and A. A. J. Marley in [2] (page 55): What are the solutions of equation (B $\Delta$ ), where the functions $F: X \times X \rightarrow X$ and $G: \Delta \rightarrow X$ are strictly increasing in each variable and $F$ is symmetric, that is

$$
F(x, y)=F(y, x), \quad(x, y) \in X^{2},
$$

and $(\mathrm{B} \Delta)$ holds for all $(x, y) \in \Delta$ and $(u, v) \in \Delta$. Equation $(\mathrm{B} \Delta)$ can be considered as a special case of the equation of generalized bisymmetry with two unknown functions (one of them is symmetric) defined on restricted domain. The following two theorems play important role in our investigations.

Theorem 2. (Maksa [4]) Let $R_{n} \subset H \subset \mathbb{R}^{2}$ be rectangles and let $R_{n} \subset R_{n+1}$ for all $n \in \mathbb{N}$. If $Q: H \rightarrow \mathbb{R}$ is a quasisum on $R_{n}$ for all $n \in \mathbb{N}$, then $Q$ is a quasisum on $\bigcup_{n=1}^{\infty} R_{n}$.

Theorem 3. (Maksa [4]) Let $R \subset \mathbb{R}^{2}$ be a rectangle and suppose that $Q: R \rightarrow \mathbb{R}$ is a $C M$ function and $Q$ is a quasisum on int $R$ (interior of $R$ ). Then $Q$ is a quasisum on $R$.

\section{The solution of equation $(\mathrm{B} \Delta)$}

Theorem 4. Let $X$ be an interval of positive length and let the set $\Delta$ be defined by (1). Suppose that the functions $F: X^{2} \rightarrow X$ and $G: \Delta \rightarrow X$ are strictly increasing in each variable and $F$ satisfies (2). Then equation (B $\Delta$ ) holds for 
all $(x, y) \in \Delta$ and $(u, v) \in \Delta$ if, and only if, there exists a $C M$ function $\alpha$ : $X \rightarrow \mathbb{R}$ and there exist real numbers $0<a, 0<d_{1}, 0<d_{2}, b$ and $c$ such that $(2 a-1) c=\left(d_{1}+d_{2}-1\right) b$ and

$$
\begin{gathered}
F(x, y)=\alpha^{-1}(a \alpha(x)+a \alpha(y)+b), \quad(x, y) \in X^{2}, \\
G(x, y)=\alpha^{-1}\left(d_{1} \alpha(x)+d_{2} \alpha(y)+c\right), \quad(x, y) \in \Delta .
\end{gathered}
$$

Proof. First we show that $F$ is a quasisum on $X^{2}$. If $c \in \operatorname{int} X$ then define the subsets $X_{1}$ and $X_{2}$ of $X$ by $X_{1}=\{x \in X \mid x \geq c\}$ and $X_{2}=X_{(c)}=\{x \in X \mid$ $x \leq c\}$. Then $X_{1} \times X_{2} \subset \Delta$, so equation (B $\Delta$ ) holds for all $(x, y) \in X_{1} \times X_{2}$ and $(u, v) \in X_{1} \times X_{2}$. With the notations $X_{11}=X_{12}=X_{1}, X_{21}=X_{22}=$ $X_{2}, F_{1}=F_{2}=F$, and $G_{1}=G_{2}=G$, by Theorem 1 , we get that there exist $C M$ functions $\gamma_{2}: F\left(X_{2}, X_{2}\right) \rightarrow \mathbb{R}, \beta_{21}: X_{2} \rightarrow \mathbb{R}$, and $\beta_{22}: X_{2} \rightarrow \mathbb{R}$ such that

$$
F(x, y)=\gamma_{2}^{-1}\left(\beta_{21}(x)+\beta_{22}(y)\right), \quad(x, y) \in X_{2}^{2} .
$$

By (2), we can assume that $\beta_{21}=\beta_{22}$, that is, there exist $C M$ functions $\gamma_{(c)}$ : $F\left(X_{(c)}, X_{(c)}\right) \rightarrow \mathbb{R}$ and $\beta_{(c)}: X_{(c)} \rightarrow \mathbb{R}$ such that

$$
F(x, y)=\gamma_{(c)}^{-1}\left(\beta_{(c)}(x)+\beta_{(c)}(y)\right), \quad(x, y) \in X_{(c)}^{2} .
$$

Let $\left(c_{n}\right): \mathbb{N} \rightarrow$ int $X$ be an increasing sequence and let $\lim _{n \rightarrow \infty} c_{n}=\sup X$. Then $X_{\left(c_{n}\right)}^{2} \subset X_{\left(c_{n+1}\right)}^{2}, n \in \mathbb{N}$, furthermore int $X^{2} \subset \bigcup_{n=1}^{\infty} X_{\left(c_{n}\right)}^{2} \subset X^{2}$.

Let $Q=F, H=X^{2}$, and $R_{n}=X_{\left(c_{n}\right)}^{2}$ for all $n \in \mathbb{N}$. Then, by Theorem 2, we have that $F$ is a quasisum on $\bigcup_{n=1}^{\infty} X_{\left(c_{n}\right)}^{2}$, so $F$ is a quasisum on $\operatorname{int} X^{2}$, too. This, by Theorem 3, implies that $F$ is a quasisum on $X^{2}$, that is, there exist $C M$ functions $\gamma: F(X, X) \rightarrow \mathbb{R}, \beta: X \rightarrow \mathbb{R}$ and $\beta^{*}: X \rightarrow \mathbb{R}$ such that $F(x, y)=\gamma^{-1}\left(\beta(x)+\beta^{*}(y)\right),(x, y) \in X^{2}$. Because of (2) here we can assume again that $\beta=\beta^{*}=\alpha$, that is,

$$
F(x, y)=\gamma^{-1}(\alpha(x)+\alpha(y)), \quad(x, y) \in X^{2} .
$$

Substituting this form of $F$ into equation $(\mathrm{B} \Delta)$ we get that

$$
\alpha \circ G(x, y)+\alpha \circ G(u, v)=\gamma \circ G\left(\gamma^{-1}[\alpha(x)+\alpha(u)], \gamma^{-1}[\alpha(y)+\alpha(v)]\right),
$$

$((x, y),(u, v)) \in \Delta \times \Delta$. Let $\Delta^{\alpha}=\left\{(x, y) \in \alpha(X)^{2} \mid x \geq y\right\}$ and define the functions $K$ and $H$ by

$$
K(\xi, \eta)=\alpha \circ G\left(\alpha^{-1}(\xi), \alpha^{-1}(\eta)\right), \quad(\xi, \eta) \in \Delta^{\alpha}
$$

and

$$
H(\lambda, \omega)=\gamma \circ G\left(\gamma^{-1}(\lambda), \gamma^{-1}(\omega)\right), \quad(\lambda, \omega) \in \Delta_{+}^{\alpha},
$$

where $\Delta_{+}^{\alpha}=\left\{x+y \mid(x, y) \in \Delta^{\alpha}\right\}$. With the functions $K$, which is strictly increasing in each variable, and $H$, equation (6) goes over into the form

$$
K(p, r)+K(q, s)=H(p+q, r+s), \quad((p, r),(q, s)) \in \Delta^{\alpha} \times \Delta^{\alpha} .
$$


By Theorem 1 in Radó-Baker [5], there exist $0<d_{1} \in \mathbb{R}, 0<d_{2} \in \mathbb{R}$, and $c \in \mathbb{R}$, such that

$$
K(\xi, \eta)=d_{1} \xi+d_{2} \eta+c, \quad(\xi, \eta) \in \Delta^{\alpha}
$$

By this form of $K$ and (7), we have $\alpha \circ G\left(\alpha^{-1}(\xi), \alpha^{-1}(\eta)\right)=d_{1} \xi+d_{2} \eta+$ $c, \quad(\xi, \eta) \in \Delta^{\alpha}$, which implies that

$$
G\left(\alpha^{-1}(\xi), \alpha^{-1}(\eta)\right)=\alpha^{-1}\left(d_{1} \xi+d_{2} \eta+c\right), \quad(\xi, \eta) \in \Delta^{\alpha},
$$

and, with $x=\alpha^{-1}(\xi)$ and $y=\alpha^{-1}(\eta)$, we get that

$$
G(x, y)=\alpha^{-1}\left(d_{1} \alpha(x)+d_{2} \alpha(y)+c\right), \quad(x, y) \in \Delta .
$$

Substituting the forms of $F$ and $G$, given by (5) and (8), into (B $\Delta$ ) we have

$$
\begin{gathered}
\gamma^{-1}\left(d_{1} \alpha(x)+d_{2} \alpha(y)+d_{1} \alpha(u)+d_{2} \alpha(v)+2 c\right)= \\
\alpha^{-1}\left(d_{1} \alpha \circ \gamma^{-1}[\alpha(x)+\alpha(u)]+d_{2} \alpha \circ \gamma^{-1}[\alpha(y)+\alpha(v)]+c\right),
\end{gathered}
$$

$((x, y),(u, v)) \in \Delta \times \Delta$. With the notations $\chi=\alpha \circ \gamma^{-1}, t=\alpha(x)+\alpha(u)$, and $s=\alpha(y)+\alpha(v),(9)$ implies that

$$
\chi\left(d_{1} t+d_{2} s+2 c\right)=d_{1} \chi(t)+d_{2} \chi(s)+c, \quad(t, s) \in \Delta_{+}^{\alpha} .
$$

Since $\chi$ is continuous a well-known argument shows that $\chi$ is differentiable, too. Thus, by (10), we have that $\chi^{\prime}\left(d_{1} t+d_{2} s+2 c\right)=\chi^{\prime}(t)=\chi^{\prime}(s)$, that is $\chi^{\prime}$ is constant on $\alpha(X)$, so there exist $0 \neq a \in \mathbb{R}$ and $b \in \mathbb{R}$ such that $\chi(s)=a s+b, s \in \alpha(X)$. Substituting this form of $\chi$ into (10) we have

$$
(2 a-1) c=\left(d_{2}+d_{1}-1\right) b
$$

It is easy to check that the functions $F$ and $G$ given by (3) and (4), where $\alpha: X \rightarrow \mathbb{R}$ is a $C M$ function and $0<a, 0<d_{1}, 0<d_{2}, b$ and $c$ are real numbers such that (11) holds, satisfy $(\mathrm{B} \Delta)$.

\section{Acknowledgement}

This research has been supported by the Hungarian National Foundation for Scientific Research (OTKA), grant No. T-030082.

\section{References}

[1] J. ACZÉL, Lectures on Functional Equations and their Applications, Academic Press, New York - London, 1966.

[2] R. D. LuCE, A. A. J. Marley, Ranked Additive Utility Representations of Gambles: Old and new Axiomatizations, The Journal of Risk and Uncertainty 30:1 (2005), 21-62.

[3] Gy. MAKSA, Solution of generalized bisymmetry type equations without surjectivity assumptions, Aequationes Math. 57 (1999), 50-74. 
[4] Gy. Maksa, Quasisums and generalized associativity, Aequationes Math. 69 (2005), 6-27.

[5] F. RAdó, J. A. BAKER, Pexider's equation and aggregation of allocations, Aequationes Math. 32 (1987), 227-239.

I. Kocsis

Faculty of Engineering University of Debrecen

H-4028 Debrecen,Ótemető u. 2-4., Hungary 